\title{
Limitation of Nocturnal ATP Import into Chloroplasts Seems to Affect Hormonal Crosstalk, Prime Defense, and Enhance Disease Resistance in Arabidopsis thaliana
}

\author{
Gudrun Schmitz, ${ }^{1}$ Thomas Reinhold, ${ }^{2}$ Cornelia Göbel, ${ }^{3}$ Ivo Feussner, ${ }^{3}$ H. Ekkehard Neuhaus, ${ }^{2}$ and \\ Uwe Conrath ${ }^{1}$ \\ ${ }^{1}$ Plant Biochemistry \& Molecular Biology Group, Department of Plant Physiology, RWTH Aachen University, Aachen, 52056, \\ Germany; ${ }^{2}$ Department of Plant Physiology, Kaiserslautern Tech, Kaiserslautern, 67663, Germany; ${ }^{3}$ Department of Plant \\ Biochemistry, Albrecht von Haller Institute for Plant Sciences, Georg August University, Göttingen 37077, Germany
}

Submitted 24 February 2010. Accepted 8 July 2010.

\begin{abstract}
When grown under short-day conditions at low light, leaves of an Arabidopsis thaliana (accession Col-0) mutant with defects in the two genes encoding plastid ATP/ADP antiporters (so-called $n t t 1-2$ null mutants) display a variety of physiological changes. These include the formation of necrotic lesions and the accumulation of hydrogen peroxide in the leaves. Here, we show that, under short-day conditions, leaves of the $n t t 1-2$ mutant display enhanced resistance to Hyaloperonospora arabidopsidis, Botrytis cinerea, and Pseudomonas syringae pv. tomato DC3000. Resistance to these pathogens was associated with constitutively elevated levels of the plant hormone salicylic acid and, eventually, jasmonic acid, and constitutive or primed activation after pathogen attack of various defense genes that are dependent on these hormones. In addition, the antagonistic crosstalk between the salicylic acid and jasmonic acid signaling pathways seems to be affected in $n t t 1-2$. Because the enhanced resistance of $n t t 1-2$ to $H$. arabidopsidis was not seen when the mutant was grown under long-day conditions, our findings argue that nocturnal ATP import into chloroplasts is crucial to keep $A$. thaliana from runaway activation of pathogen resistance.
\end{abstract}

In plants, resistance to pathogens depends on the timely activation of defense. This has been shown for the so-called genefor-gene resistance (Boch et al. 1998; Yu et al. 1998) and various types of induced resistance (Conrath et al. 2002, 2006). Timely activation of defense, in turn, depends on the well-balanced supply of metabolites, hormones, energy, and redox power (Berger et al. 2007; Bolton 2009). Over the past few years, it has been appreciated that primary metabolism and retrograde signaling from organelles play important roles in the overall defense capacity of plants (Bolton 2009; Jung and Chory 2009). However, except for certain hormones, little is known about the identity of metabolites and metabolic pathways by which organelles would modulate a plant's defense capacity (Berger et al. 2007; Bolton 2009; Jung and Chory 2009).

Both positive and negative crosstalk between hormone signaling pathways have been reported in plants (Beckers and Spoel 2006; Koornneef and Pieterse 2008). Probably the best example for negative crosstalk between plant hormones is the interaction between salicylic acid (SA) and jasmonic acid/

Corresponding author: U, Conrath;

E-mail: uwe.conrath@bio3.rwth-aachen.de ethylene (JA/ET) signaling in Arabidopsis thaliana (Beckers and Spoel 2006; Koornneef and Pieterse 2008; Leon-Reyes et al. 2009). It is widely accepted that, in this plant, the SA pathway mainly regulates the defense to biotrophic pathogens while necrotrophic pathogens and insects are more sensitive to defense responses induced by JA and ET (Thomma et al. 1998; Kessler and Baldwin 2002; Ton et al. 2002; De Vos et al. 2006; Abe et al. 2008). There also is ample evidence that SA and JA signaling pathways are mutually antagonistic (Beckers and Spoel 2006; Koornneef and Pieterse 2008; Koornneef et al. 2008). This negative crosstalk between pathways is assumed to help fine tune and, eventually, boost a plant's defense response to a given type of attacking pathogen (Reymond and Farmer 1998).

Various important metabolic pathways (e.g., synthesis of starch, fatty acids, SA, and, partially, JA) are localized to plastids (Neuhaus and Wagner 2000); therefore, these organelles are excellent candidates for cellular compartments with an important role in the coordination of defense. This assumption is supported by our earlier work, in which we showed that antisensing the plastid ATP/ADP transporter in potato tubers primes pathogen-activated defense responses and enhances the resistance to bacterial and fungal pathogens (Linke et al. 2002; Conrath et al. 2003). However, the role of chloroplast nucleotide transporters (NTT), the signaling function of nucleotides, and the impact of well-balanced nocturnal ATP import into chloroplast for the overall pathogen defense of photoautotrophic plant tissue have remained elusive.

NTT are found in all lower and higher plants analyzed thus far (Linka et al. 2003). They are localized to the inner membrane of the plastid envelope (Neuhaus et al. 1997) and catalyze the import of ATP from within the cytoplasm and the export of ADP and orthophosphate off the plastid in a well-balanced 1:1 stoichiometry (Trentmann et al. 2008). In A. thaliana, two isoforms of plastid ATP/ADP transporter, referred to as NTT1 and NTT2, mediate ATP/ADP antiport over the inner membrane of the plastid envelope (Reiser et al. 2004). To elucidate the physiological relevance of chloroplast NTT, Reiser and associates (2004) generated double knockout A. thaliana mutants lacking both functional NTT genes (so-called nttl-2 null mutants). These mutants were used to analyze molecular, biochemical, and physiological consequences of the absence of NTT1 and NTT2 (Reinhold et al. 2007). Under short days at low light, these consequences include the activation of various genes with a role in redox homeostasis, accumulation of protoporphyrin IX, light-induced $\mathrm{H}_{2} \mathrm{O}_{2}$ accumulation, and the 
appearance of necrotic lesions on the leaves (Reinhold et al. 2007). Because lesion formation has often been associated with the induction of disease resistance, ntt1-2 mutants are excellent tools to investigate the phytopathological relevance of i) chloroplast NTT proteins, ii) the ATP/ADP antiport over the chloroplast envelope, and iii) altered nocturnal starch degradation. Here, we show that, in A. thaliana, limited ATP/ADP supply to chloroplasts affects hormonal crosstalk, leads to primed activation of defense responses after infection, and causes resistance to pathogens with different lifestyles.

\section{RESULTS}

Enhanced resistance to Hyaloperonospora arabidopsidis in $n t t 1-2$ under short-day conditions.

To investigate whether $n t t l-2$ has higher pathogen resistance under short-day than long-day conditions, the ntt1-2 mutant and corresponding wild-type plants (accession Col-0) were grown under both conditions. Three-week-old seedlings of both genotypes of plant were spray inoculated with a conidiospore suspension of the biotrophic oomycete Hyaloperonospora arabidopsidis (isolate Noco) (Rethage et al. 2000) and assayed for pathogen propagation by trypan blue staining (Fig. 1A). Under long-day conditions, $H$. arabidopsidis formed intercellular hyphae in the leaves of both genotypes after one week (Fig. 1A). Under short-day conditions, H. arabidopsidis development was even further advanced in wild-type plants, as was evident by the formation of conidiophores that emerged from leaf surfaces (Fig. 1A). In contrast, the ntt1-2 mutant displayed a trailing necrosis resistance response (Mauch-Mani and Slusarenko 1994) one week after infection under short-day conditions (Fig. 1A), which finally stopped hyphal growth of the pathogen.

Determination of the number of spores released from $H$. arabidopsidis-infected leaves at the seventh day after infection confirmed that there has been no obvious difference in pathogen development between wild-type and nttl-2 plants under long-day conditions (Fig. 1B). However, when grown under short-day conditions, $H$. arabidopsidis released nearly 10 times fewer spores from leaves of the inoculated $n t t 1-2$ mutant (Fig. 1B).

\section{H. arabidopsidis-induced defense gene expression is primed in short-day-grown $n t t 1-2$.}

To identify molecular events associated with the enhanced resistance of the ntt1-2 mutant to $H$. arabidopsidis under shortday conditions, we next assayed the expression of selective marker genes for the activation of the SA or JA pathways. These two hormones have been associated with the induction of resistance to pathogens in A. thaliana (Thomma et al. 1998). Under short-day conditions and before infection, there was slightly enhanced basal expression of $P R 1, P R 2$, and $P R 5$ in $n t t 1-2$ when compared with wild-type plants (Fig. 2A). After inoculation with $H$. arabidopsidis, expression of the three defense genes was activated in both genotypes of plant but it was faster in $n t t 1-2$ than in the wild type (Fig. 2A). In the latter, expression of $P R 1, P R 2$, and $P R 5$ was high at the seventh day postinoculation. At this time, expression of the three $P R$ genes was downregulated again in the $n t t 1-2$ mutant (Fig. 2A).

Similar findings were made when the expression of the JAresponsive genes $P R 3, P R 4$, and $P D F 1.2$ was measured (Fig. 2B). Basal expression of the three genes was slightly higher in nttl-2 plants than in the wild type when both genotypes of plant were grown under short-day conditions (Fig. 2B). After inoculation with $H$. arabidopsidis, activation of $P R 3$ and $P R 4$ was faster in $n t t 1-2$ than in the wild type (Fig. 2B). In the latter, expression of $P R 3$ and $P R 4$ was high at the seventh day postinoculation with $H$. arabidopsidis when their activation was downregulated again in $n t t 1-2$ (Fig. 2B). Basal PDF1.2 expression also was slightly augmented in $n t t l-2$ before infection (Fig. 2B). After inoculation with $H$. arabidopsidis, activation of PDF 1.2 was maximal at the second day postinfection in both genotypes of plant and declined afterward. It also was
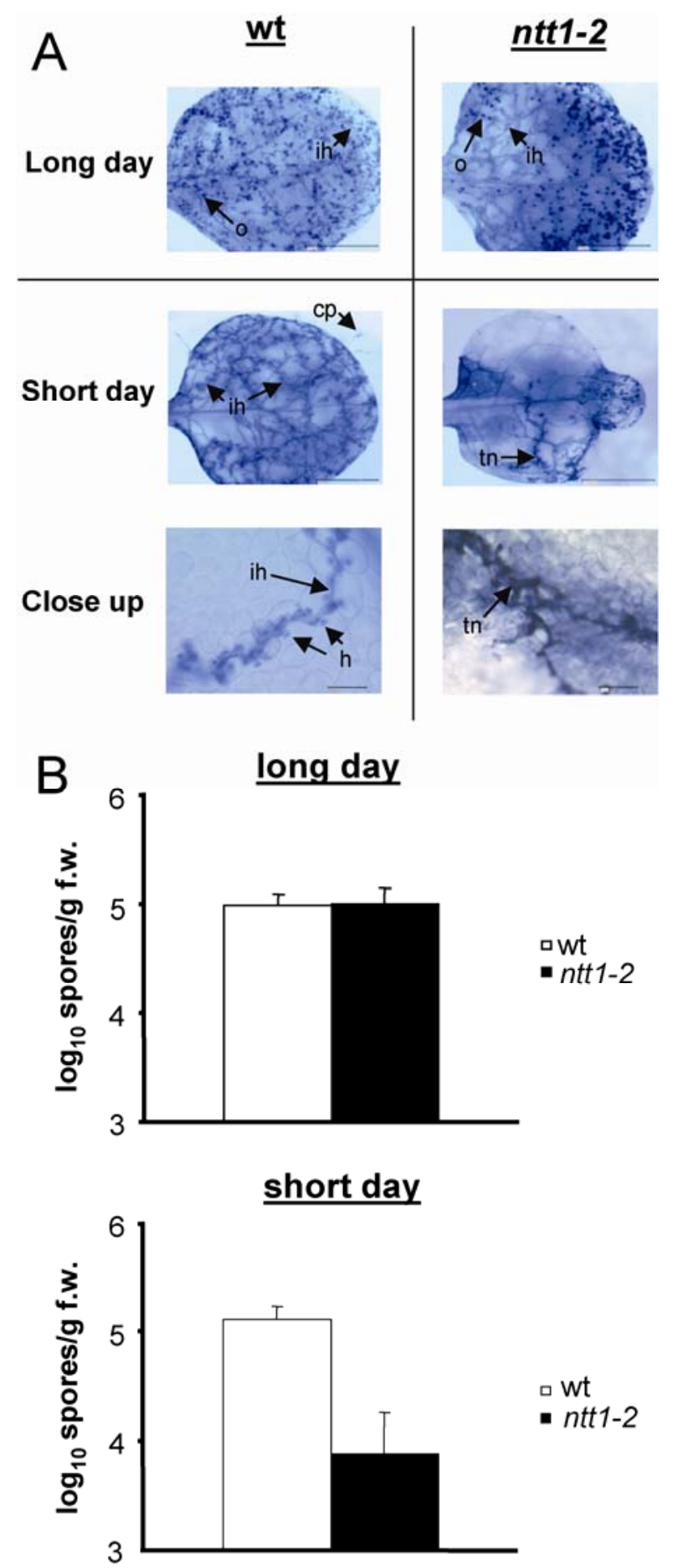

Fig. 1. Development of Hyaloperonospora arabidopsidis on wild-type (wt) and $n t t 1-2$ seedlings. A, Plants were grown under long-day or shortday conditions for 3 weeks. Seedlings were inoculated with conidiospores of $H$. arabidopsidis, and growth of the oomycete assayed by trypan blue staining after 1 week; ih, intercellular hypha, o, oospore, cp, conidiophores, tn, trailing necrosis, h, haustorium. B, Same experimental setup as in A, except that spores were washed from infected leaves with water and an aliquot of spore suspension was counted under a microscope. Values shown are means \pm standard deviation $(n=4)$. 
more robust in the $n t t 1-2$ mutant than in the wild type at all time points tested (Fig. 2B). Together, the results in Figure 2 demonstrate that the expression of genes in both the SA and JA pathway is primed in $n t t l-2$ when grown under short-day conditions and infected with $H$. arabidopsidis.

\section{SA and JA levels in $\boldsymbol{n t t 1 - 2}$ before and after inoculation with $H$. arabidopsidis.}

To investigate whether the primed activation of genes in the SA and JA pathway by $H$. arabidopsidis in short-day-grown nttl-2 plants (Fig. 2) is associated with enhanced basal levels or augmented induction after inoculation of SA and JA, the levels of the two hormones were determined in nttl-2 and wild-type seedlings before and after their inoculation with $H$. arabidopsidis. Short-day-grown nttl-2 plants, in contrast to the wild type, have constitutively enhanced SA levels before inoculation (Fig. 3). These SA levels were only slightly, if at all, further enhanced after inoculation with $H$. arabidopsidis in $n t t 1-2$, whereas they increased considerably from low basal levels after inoculation with $H$. arabidopsidis in wild-type plants (Fig. 3).

In contrast to SA, the basal level of JA was not clearly enhanced in $n t t l-2$ before inoculation (Fig. 3). Although a faint enhancement was seen in all experiments performed, this enhancement was not significant. After infection with $H$. arabidopsidis, endogenous JA levels remained at a low basal level in both the wild type and nttl-2 until at least the fourth day postinoculation. They seemed to be elevated in both genotypes of plant at the seventh day after infection, yet this increase was not significant (Fig. 3).

\section{Enhanced resistance of $n$ tt1-2 to Botrytis cinerea.}

To further characterize the resistance response of $n t t 1-2$ plants under short-day-conditions, we extended our analysis to pathogens with other lifestyles. The necrotrophic gray mold fungus Botrytis cinerea was chosen as the second pathogen to inoculate short-day-grown plants of the nttl-2 mutant and the wild type. Leaves of 5- to 6-week-old $n t t 1-2$ and wild-type
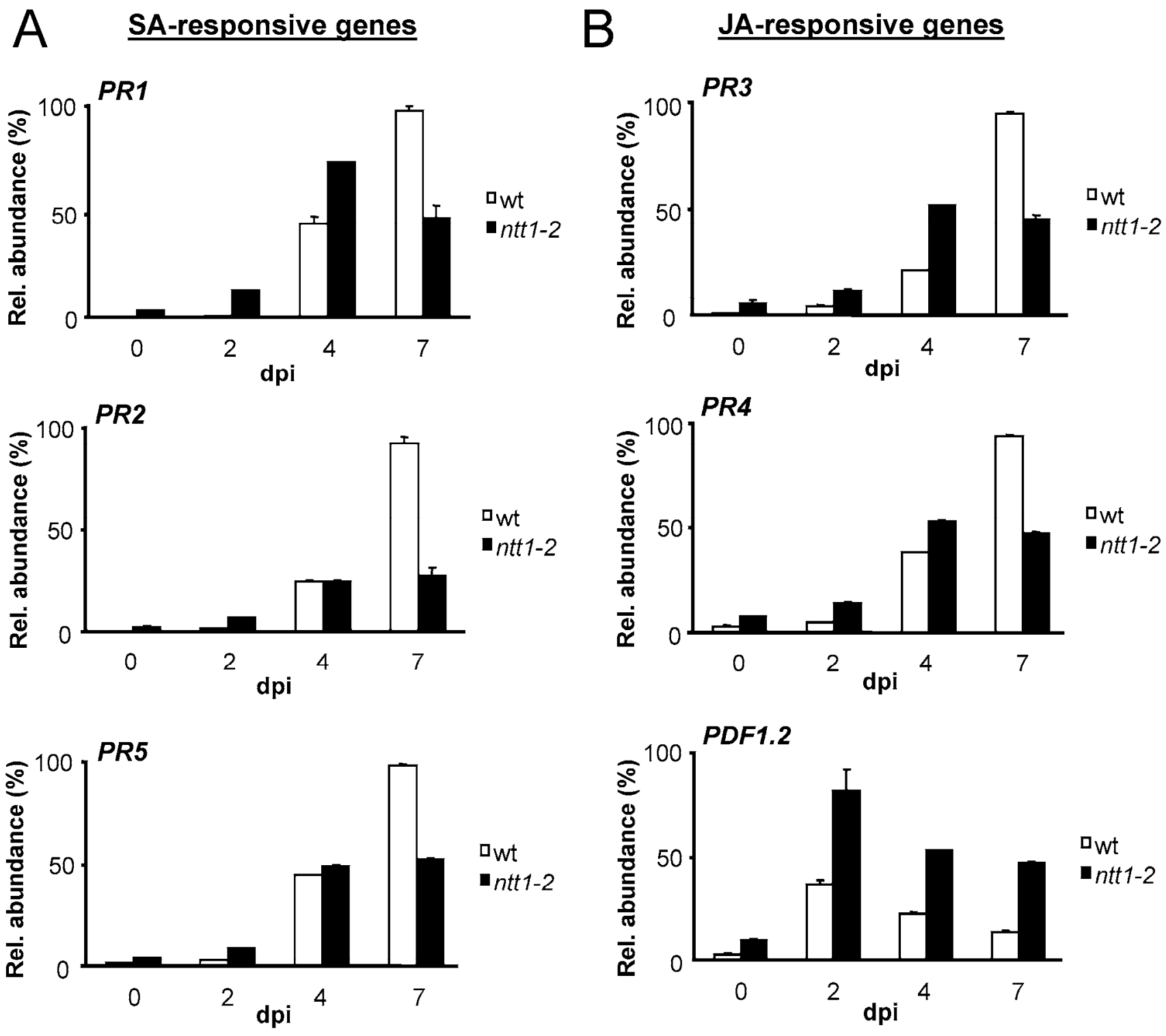

Fig. 2. Expression of marker genes of the salicylic acid (SA) and jasmonic acid (JA) pathway in short-day-grown wild-type (wt) and $n t t 1-2$ seedlings before and after infection with Hyaloperonospora arabidopsidis. Three-week-old seedlings were harvested before or at various time points after inoculation with $H$. arabidopsidis. Leaves were used for RNA extraction and quantitative reverse-transcription polymerase chain reaction analysis to examine the abundance of $P R 1, P R 2, P R 3, P R 4, P R 5$, and PDF1.2 transcript normalized to ACTIN2. Values shown are means \pm standard deviation $(n=3)$; dpi $=$ days postinfection. Shown is the result of one of three experiments with similar results. 
plants were inoculated with the gray mold fungus (Zimmerli et al. 2001) by deposition of 5- $\mu$ l droplets containing $5 \times 10^{5}$ spores $\mathrm{ml}^{-1}$ of $B$. cinerea. At the third day postinoculation, the surface of $B$. cinerea-induced necroses on the infected leaves was much smaller in nttl-2 than it was in the wild type (Fig. 4). Cytological investigation of infected leaves by trypan blue staining revealed that fungal hyphae grew concentrically from the site of inoculation in both genotypes of plant but the area of infected leaf tissue was smaller in $n t t 1-2$ when compared with the wild type (Fig. 4). Thus, the macroscopic symptoms reflect the intensity of infection in $n t t l-2$ and the wild type.

Defense gene activation by $B$. cinerea is primed in $n t t 1-2$.

As a next step in the characterization of the resistance response of $n t t 1-2$, we assayed the expression of the SA-inducible $P R 1, P R 2, P R 3$, and PDF1.2 defense genes in leaves of $n t t 1-2$ and the wild type before and after inoculation with $B$. cinerea. There was enhanced basal expression of $P R 1$ and $P R 2$ in $n t t 1-2$ (Fig. 5A). After B. cinerea infection, expression of the two genes was induced in both genotypes but the activation of $P R 1$ and $P R 2$ was more robust in the ntt1-2 mutant (Fig. $5 \mathrm{~A})$.

Similar to $P R 1$ and $P R 2$, basal expression of the $P R 3$ and $P D F 1.2$ genes also was faintly enhanced in $n t t 1-2$ in the absence of pathogen attack (Fig. 5B). After infection with $B$. cinerea, both $P R 3$ and $P D F 1.2$ were induced in both $n t t 1-2$ and the wild type, and their expression was higher in wild-type than in $n t t 1-2$ plants (Fig. 5B).

\section{SA and JA levels in B. cinerea-inoculated ntt1-2 and the wild type.}

As a next step in the characterization of the resistance response in the nttl-2 mutant, SA and JA levels were measured in $n t t 1-2$ and the wild type before and after inoculation with $B$. cinerea. Similar to A. thaliana seedlings (Fig. 3) before infection, the endogenous levels of SA were higher in adult $n t t 1-2$ plants than in the wild type whereas basal JA levels were not significantly different between the two genotypes of plant (Fig. $6)$. After inoculation of leaves with $B$. cinerea, SA levels rose in both the wild type and $n t t 1-2$. They were high in $n t t 1-2$ at the second and third day postinoculation. At these time points, SA levels seemed to be downregulated again in wild-type leaves (Fig. 6). JA levels also increased after $B$. cinerea infection in both genotypes of plant, with a tendency for higher levels in the wild type than in $n t t l-2$ at all time points tested (Fig. 6).

\section{Enhanced resistance to Pseudomonas syringae pv. tomato in $n t t 1-2$ under short-day conditions.}

As a third pathogen to characterize the resistance response in $n t t 1-2$ under short-day conditions, we choose the hemibiotrophic bacterial pathogen Pseudomonas syringae pv. tomato (strain DC3000). Wild-type and nttl-2 plants were inoculated on their leaves by dipping into a suspension of $P$. syringae pv. tomato DC3000. At various days after inoculation, bacteria were reisolated from infected leaves transferred to agar plates, and developing colonies counted. The titer of $P$. syringae pv. tomato DC3000 increased with time in both nttl-2 and the wild type (Fig. 7A). However, in the ntt1-2 mutant, $P$. syringae pv. tomato DC3000 multiplication was considerably lower than in wild-type plants (Fig. 7A).

\section{$P$. syringae pv. tomato DC3000-induced}

defense gene activation is primed in $\mathbf{n t t} \mathbf{1 - 2}$.

Next, we decided to assay the activation of a reliable marker gene for the SA pathway $(P R l)$ and a representative one for the JA signaling route $(P R 4)$ after inoculation of $n t t 1-2$ and wild-type plants with $P$. syringae pv. tomato DC3000. Enhanced basal expression of $P R I$ (Figs. 2A, 5A, and 7B) in shortday-grown $n t t 1-2$ plants is further enhanced upon $P$. syringae pv. tomato DC3000 infection (Fig. 7B). The enhancement was seen already at the first day post-bacterial inoculation when $P R 1$ expression was still very low in the inoculated wild type (Fig. 7B). In the latter, $P R 1$ activation was high at the third day after infection, when $P R I$ activation was already downregulated again in the $n t t 1-2$ mutant (Fig. 7B).

Similar findings were made when the expression of PR4 was assayed. Again, the slightly enhanced level of basal expression in $n t t l-2$ (Figs. 2B and 7B), although at overall low levels, is more robust at the first day after inoculation in the $n t t 1-2 \mathrm{mu}-$ tant than in the wild type whereas, in the latter, $P R 4$ activation is high at the third day post-bacterial infection (Fig. 7B). Thus, the nttl-2 mutant is primed for more robust activation of both the SA-dependent $P R I$ and JA-dependent $P R 4$ gene after infection with $P$. syringae pv. tomato DC3000.

\section{DISCUSSION}

In plants, coordinated activation of pathogen defense depends on the well-balanced supply of metabolites, hormones, energy, and redox power (Berger et al. 2007; Bolton 2009; Jung and Chory 2009). Here, we showed that, under short-day conditions, the A. thaliana ntt1-2 mutant has enhanced resistance to a biotrophic, hemibiotrophic, and necrotrophic pathogen (Figs. 1,4 , and 7A). Because the augmented resistance to $H$. arabidopsidis, B. cinerea, and P. syringae pv. tomato DC3000 cannot be seen when $n t t 1-2$ is grown under long-day conditions (Fig. 1A) (data not shown), sufficient nocturnal ATP supply to the chloroplasts of these mutants by glycolytic starch degradation or nocturnal ATP import into the chloroplasts seem to be
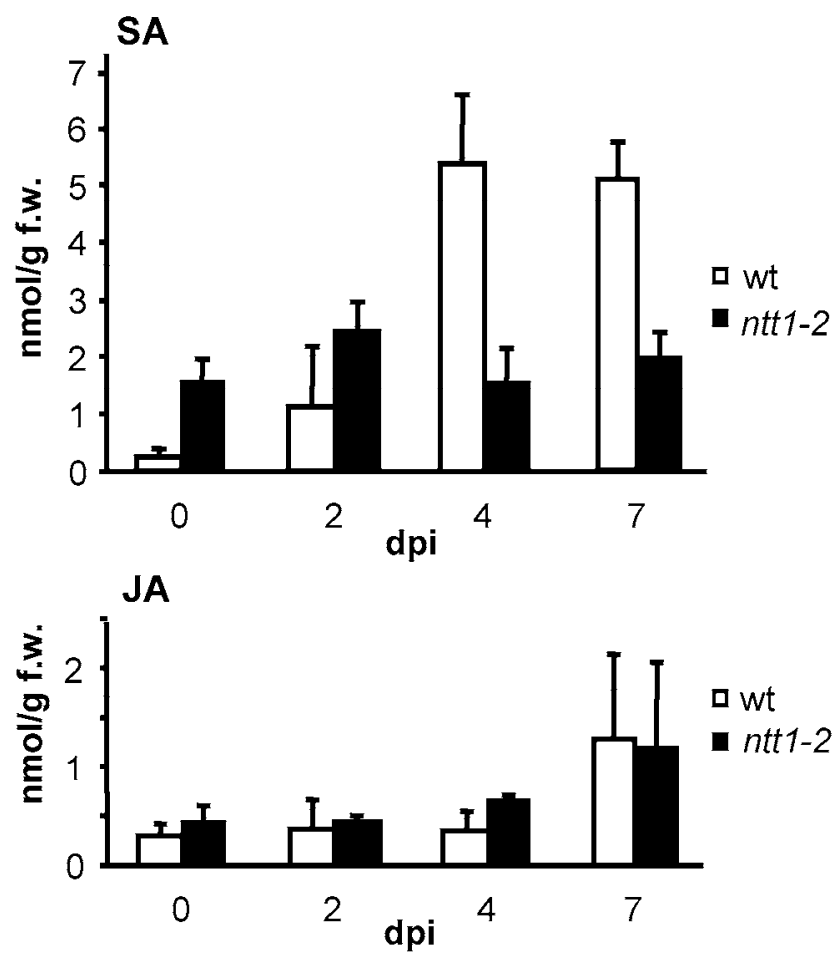

Fig. 3. Levels of salicylic acid (SA) and jasmonic acid (JA) in short-daygrown $n t t 1-2$ and wild-type (wt) plants before and after infection with Hyaloperonospora arabidopsidis. Leaves of seedlings were harvested before and at various time points after inoculation with $H$. arabidopsidis and assayed for SA and JA levels by gas chromatography. The experiment was done three times with two biological replicates; dpi = days postinfection. Values shown are means \pm standard deviation $(n=6)$. 
crucial processes that keep A. thaliana from runaway activation of broad-spectrum disease resistance in the absence of pathogens. A similar conclusion was drawn by Reinhold and associates (2007), who concluded that nocturnal ATP import into chloroplast is needed for controlled biosynthesis of chlorophyll, avoidance of spontaneous lesion formation, and prevention of photooxidative damage (Reinhold et al. 2007). These authors also demonstrated that $n t t 1-2$ plants have stimulated glycolytic starch degradation when compared with wildtype plants (Reinhold et al. 2007). wt
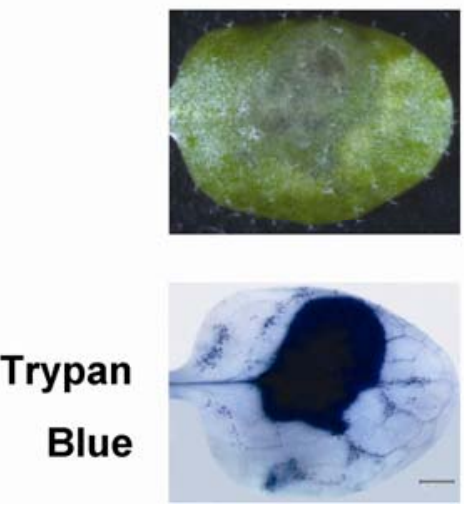

$\underline{n t t 1-2}$
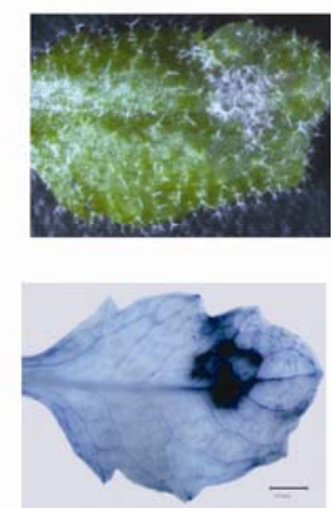

Fig. 4. Botrytis cinerea-induced lesions on wild-type (wt) and ntt1-2 plants. Plants were grown under short-day conditions for 5 to 6 weeks and then inoculated with spores of $B$. cinerea. After 3 days, infected leaves were photographed under a stereomicroscope to document disease lesions. Then, infected leaves were subjected to trypan blue staining (scale bar = $1,000 \mu \mathrm{m})$. Note the enhanced number of trichomes on the ntt1-2 mutant. The experiment was done three times with similar results.
Under short-day conditions, nttl-2 displayed light-induced $\mathrm{H}_{2} \mathrm{O}_{2}$ accumulation and constitutive expression of genes for copper/zinc superoxide dismutase 2 and ascorbate peroxidase 1 (Reinhold et al. 2007). Thus, it is likely that the cellular redox balance is attenuated in short-day-grown $n t t 1-2$. Because redox regulation plays an important role in the control of the key regulatory protein NPR1 in the SA signaling pathway (Dong 2004) and because redox modulation also has an essential role in the SA-mediated attenuation of the JA signaling pathway (Koorneef et al. 2008), the reported change in cellular redox state in $n t t 1-2$ (Reinhold et al. 2007) might be the cause for affected crosstalk between the SA and JA pathways in this mutant (Figs. 2 and 7B). The lack of negative crosstalk between SA and JA becomes obvious by the simultaneous rather than mutually exclusive activation of genes in the two hormone pathways after attack by $H$. arabidopsidis (Fig. 2) or $P$. syringae pv. tomato (Fig. 7B). In the interaction with $B$. cinerea, $n t t 1-2$ is characterized by enhanced SA levels and $P R 1$ and $P R 2$ expression and, at the same time, decreased levels of JA and $P R 3$ and $P D F 1.2$ activation when compared with the wild type (Fig. 5); therefore, negative crosstalk between the SA and JA pathways seems to be active in the ntt1-2-B. cinerea interaction, at least to a certain extent.

SA levels and, perhaps, slightly, JA levels as well, are enhanced in uninfected young and adult ntt $1-2$ plants grown under short-day conditions (Figs. 3 and 6). The assumption of a faint though statistically insignificant increase in JA levels in uninfected nttl-2 plants would be consistent with the low constitutive activation of JA-responsive genes in $n t t 1$-2 (Figs. 2, 5, and 7B). Regardless, the increase in hormone levels is mostly associated with only faint activation of SA- and JA-responsive genes before inoculation of the plants with a pathogen (Figs. 2, 5, and 7B). After infection of $n t t 1-2$ with $H$. arabidopsidis, $B$. cinerea, or $P$. syringae pv. tomato under short-day conditions,

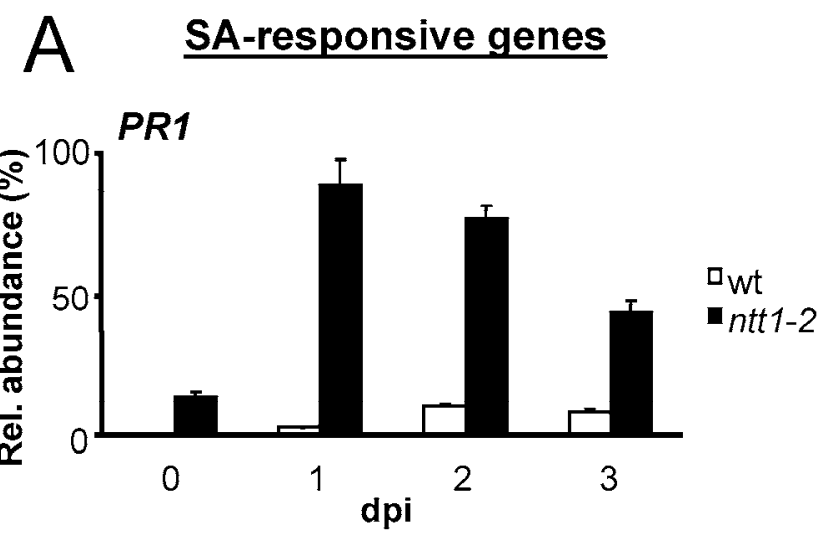

B

\section{$\underline{\text { JA-responsive genes }}$}

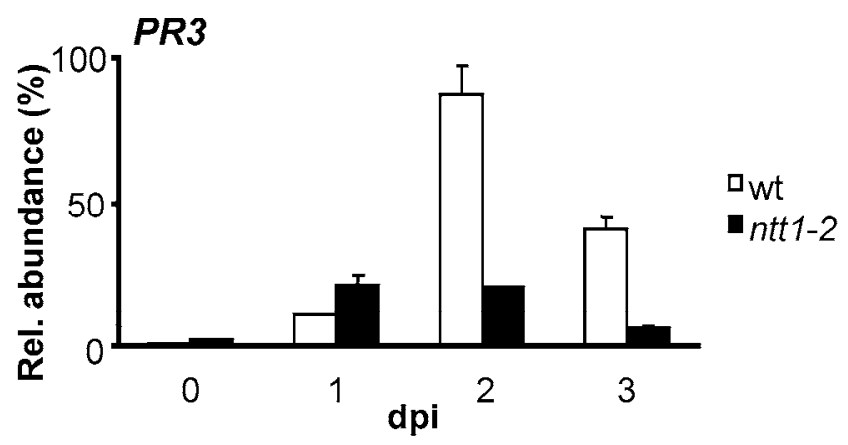

PDF1.2

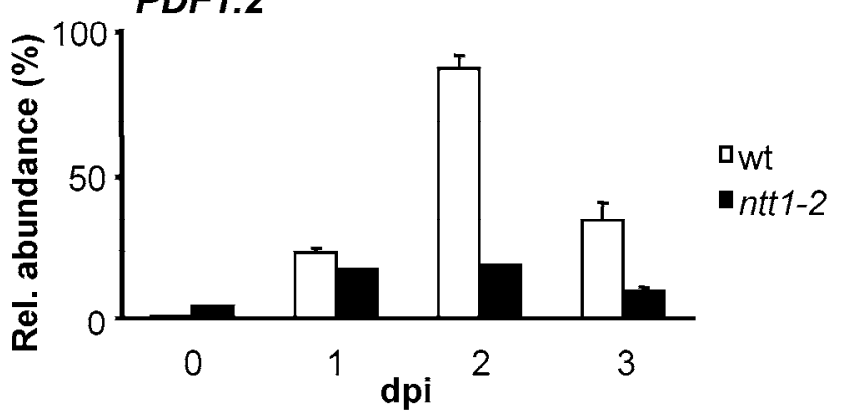

Fig. 5. Expression of defense genes in the salicylic acid (SA) or jasmonic acid (JA) pathway in short-day grown wild-type (wt) and $n t t 1$-2 plants before and after infection with Botrytis cinerea. The 5- to 6-week-old plants were harvested before or at various times after inoculation with the gray mold fungus. Leaves were used for RNA extraction and quantitative reverse-transcription polymerase chain reaction analysis to determine the abundance of $P R 1, P R 2$, $P R 3$, and PDF1.2 transcript normalized to ACTIN2. The values shown are means \pm standard deviation $(n=3)$; dpi $=$ days postinfection. Shown is the result of one of three independent experiments with similar results. 
the activation of both types of genes is faster or more robust in $n t t 1-2$ than in the wild type (Figs. 2, 5, and 7B). Thus, the enhanced levels of SA and, eventually, JA in the nttl-2 mutant are associated with low-level activation of SA- and JA-responsive genes and with the induction of a primed state for potentiated activation of defense genes in the JA and SA pathways after attack by pathogens with different lifestyles, and this is associated with enhanced resistance to these pathogens (Figs. 1,4 , and 7A). These results are reminiscent of our earlier findings, in which antisensing the plastid ATP/ADP transporter in potato tubers primed the tissue for enhanced defense gene activation and resistance to Pectobacterium atrosepticum and $\mathrm{Al}$ ternaria solani (Linke et al. 2002; Conrath et al. 2003). However, in contrast to A. thaliana leaves (Figs. 3 and 6), the antisense-induced priming in tubers was not associated with enhanced levels of SA in these organs (Linke et al. 2002). In the tubers, priming could be due to enhanced sensitivity to SA.

Extracellular ATP has recently been shown to play a critical regulatory role in defense signaling and disease resistance in plants (Song et al. 2006; Foresi et al. 2007; Wu et al. 2008; Chivasa et al. 2009). Whether cytoplasmic ATP that accumulates to high levels because of decreased import into chloroplasts in $n t t 1-2$ plants has a signaling role similar to extracellu-
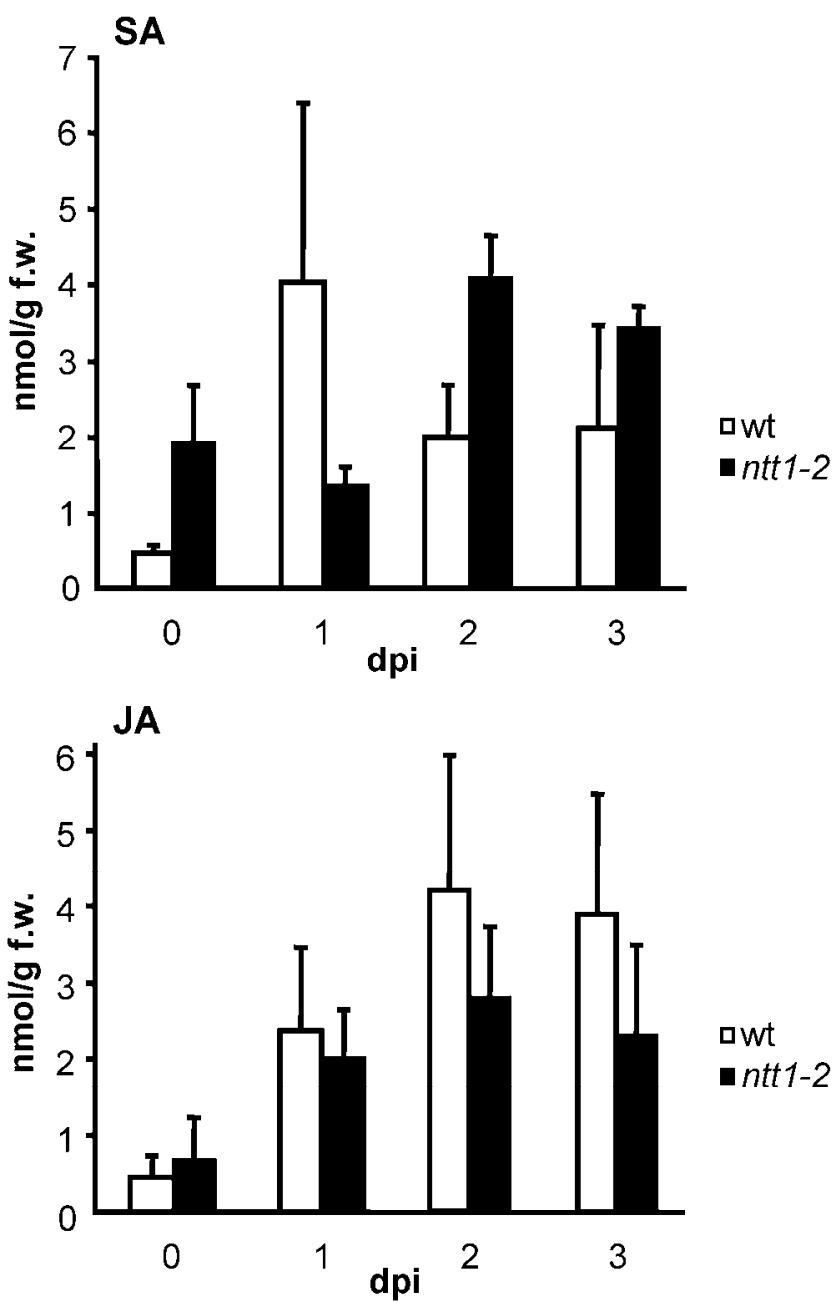

Fig. 6. Salicylic acid (SA) and jasmonic acid (JA) levels in ntt1-2 and wild-type (wt) plants under short-day conditions before and after Botrytis cinerea infection. Leaves of both genotypes of plant were harvested before and at various times after inoculation with $B$. cinerea and assayed for SA and JA levels. The experiment was done three times with two biological replicates; dpi $=$ days postinfection. Values shown are means \pm standard deviation $(n=6)$. lar ATP, or whether cytoplasmic accumulation of ATP feeds back to enhanced levels of regulatory ATP in the apoplast, remains to be determined.

\section{MATERIALS AND METHODS}

Plant growth.

A. thaliana wild-type (accession Col-0) and ntt1-2 mutant plants (in Col-0 background) (Reiser et al. 2004) were grown on soil, either at $8 \mathrm{~h}$ of light and $16 \mathrm{~h}$ of darkness (short-day conditions) or $14 \mathrm{~h}$ of light and $10 \mathrm{~h}$ of darkness (long-day conditions) at $20^{\circ} \mathrm{C}$ and 60 to $70 \%$ relative humidity.

\section{Inoculation of plants with pathogens and evaluation of pathogen development.}

H. arabidopsidis. For inoculation with $H$. arabidopsidis, 3week-old seedlings of both $A$. thaliana genotypes were spray inoculated with a conidiospore suspension $\left(3 \times 10^{4}\right.$ spores $\mathrm{ml}^{-1}$ of water) of $H$. arabidopsidis (isolate Noco). Seven days after
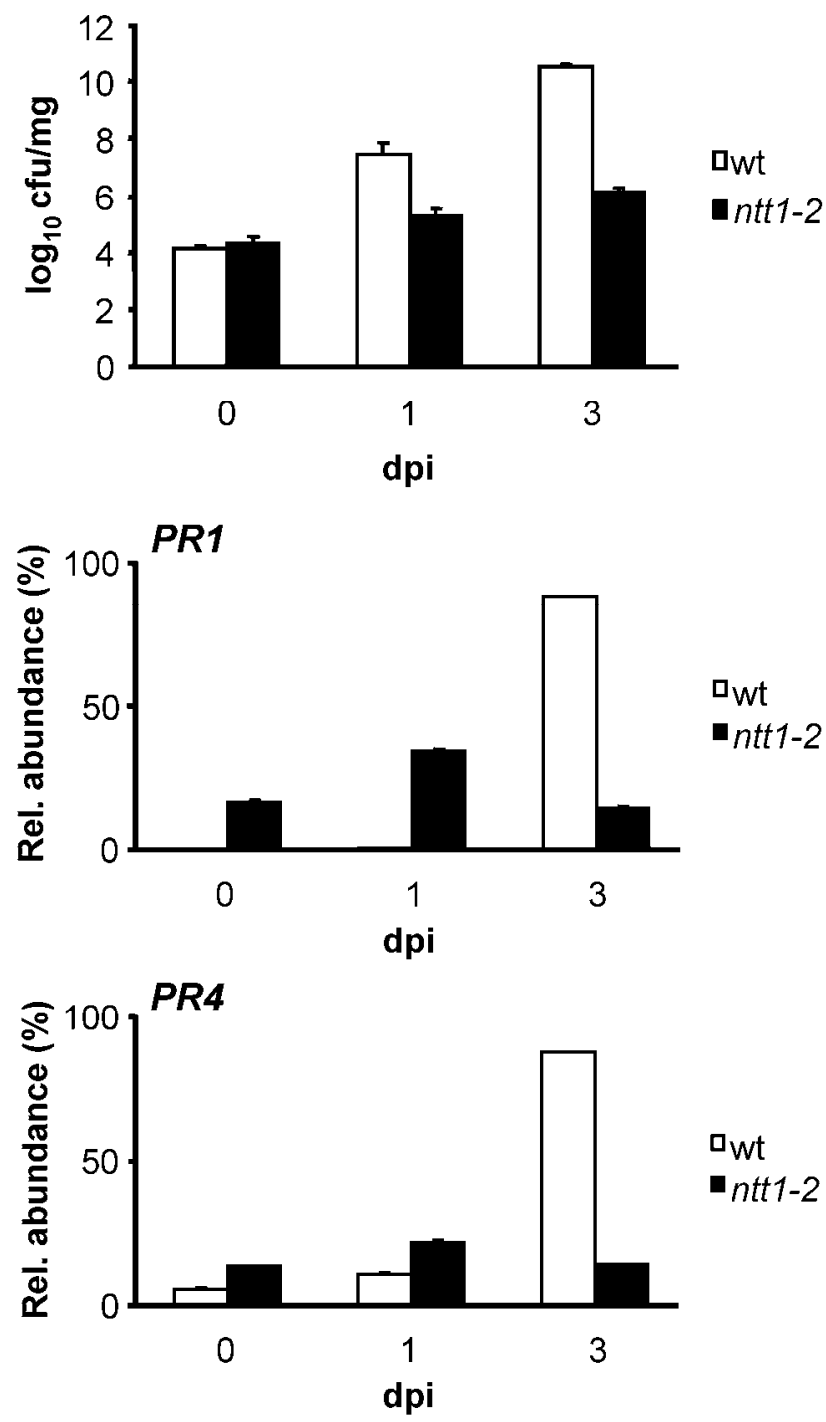

Fig. 7. Growth of Pseudomonas syringae pv. tomato and expression of defense marker genes before and after inoculation with $P$. syringae pv. tomato of short-day-grown ntt1-2 and wild-type (wt) plants. Three-weekold plants were infected by dipping leaves into a suspension of $P$. syringae pv. tomato $\left(5 \times 10^{8} \mathrm{CFU} \mathrm{ml} \mathrm{m}^{-1}\right)$. At the indicated times, leaves were harvested and analyzed for the amount of bacteria; dpi = days postinfection. Values shown are means \pm standard deviation $(n=4)$. Shown is the result of one of three independent experiments with similar results. 
inoculation, propagation of pathogen growth was assayed in leaves by trypan blue staining.

Alternatively, the number of spores released from $H$. arabidopsidis-infected leaves was determined at the seventh day after inoculation. For this, $100 \mathrm{mg}$ of infected leaf material was transferred to $1 \mathrm{ml}$ of water in an Eppendorf tube. The tube was thoroughly shaken on a Vortex mixer to release conidiospores from the infected leaf tissue. Conidiospore number was determined by counting in an aliquot of suspension in Neubauer's improved chamber.

B. cinerea. B. cinerea (strain BMM1) was grown as described (Zimmerli et al. 2001). For inoculation with the gray mold fungus, leaves of 5- to 6-week-old nttl-2 and wild-type plants were inoculated by deposition of $5-\mu 1$ droplets containing $5 \times 10^{5}$ spores $\mathrm{ml}^{-1}$ of $B$. cinerea. At 3 days after inoculation, photographs were taken from $B$. cinerea-infected leaves. Then, leaves were subjected to trypan blue staining to visualize fungal infection structures.

P. syringae $p v$. tomato. Leaves of wild-type plants and the ntt1-2 mutant were dip inoculated on their leaves with $P$. syringae pv. tomato (strain DC3000) (Tornero and Dangl 2001). At various days after inoculation, one infected leaf was harvested per plant. Three leaves were collected in Eppendorf tubes containing $1 \mathrm{ml}$ of $10 \mathrm{mM} \mathrm{MgCl} 2$ and $0.02 \%$ (vol/vol) Silwet L-77 and homogenized with a pestle. After centrifugation of the homogenate at $12,000 \times g$ in a minifuge, the supernatant was transferred to a fresh tube. An aliquot of serial dilutions of supernatant was transferred to agar plates and incubated at $28^{\circ} \mathrm{C}$ for $48 \mathrm{~h}$, and developing colonies counted.

\section{Trypan blue staining.}

A. thaliana leaves were boiled for $3 \mathrm{~min}$ in lactophenol blue solution (10 $\mathrm{g}$ of phenol, $20 \mathrm{mg}$ of trypan blue, $10 \mathrm{ml}$ of lactic acid, $10 \mathrm{ml}$ of glycerol, and $10 \mathrm{ml}$ of water) and incubated overnight. Leaves were then destained in a solution of chloral hydrate (50 $\mathrm{g}$ of chloral hydrate in $20 \mathrm{ml}$ of water). Leaves were subjected to microscopical evaluation in $50 \%$ (vol/vol) glycerol.

\section{RNA isolation and determination of gene expression.}

Total RNA was isolated from leaves using TRI Reagent (Molecular Research Center, Cincinatti, OH, U.S.A.). Total RNA $(1 \mu \mathrm{g})$ was incubated with 1 unit of DNaseI (Fermentas, St. Leon-Rot, Germany) in a total volume of $10 \mu \mathrm{l}$ at $37^{\circ} \mathrm{C}$ for $30 \mathrm{~min}$. After inactivation of DNaseI by heating to $70^{\circ} \mathrm{C}$ for 15 min, RNA was reverse transcribed using 200 units of Revert Aid M-MuLV reverse transcriptase (Fermentas) and $50 \mu \mathrm{M}$ random nanomer primers in a sample volume of $20 \mu \mathrm{l}$. Diluted cDNA was used as template for real-time quantitative reversetranscription polymerase chain reaction (qRT-PCR) with an ABI PRISM 7000 sequence detector system (Applied Biosystems, Darmstadt, Germany). Each $10-\mu 1$ reaction mix contained $5 \mu \mathrm{l}$ of SYBR Green Master mix (Applied Biosystems) and $0.3 \mathrm{mM}$ (final concentration) gene-specific primers. Real-time DNA amplification was analyzed using the ABI PRISM 7000 SDS 1.0 software (Applied Biosystems). The cycle at the threshold value was set constant throughout the study and corresponded to the log linear range of PCR amplification. The normalized amount of target reflected the relative amount of target transcripts with respect to the endogenous reference gene ACTIN2. To detect and exclude nonspecific amplicons, the melting curves of all PCR products were analyzed, all final products were visualized by agarose gel electrophoresis to ensure amplification of a single product of the correct size, and products were fully sequenced. Results of qRT-PCR analyses were verified by at least three independent experiments, each with three biological replicates (means + standard deviation, $n=$ 3 biological replicates).

\section{Determination of SA and JA levels.}

Phytohormones were extracted and analyzed as described by Stumpe and associates (2005). After gas chromatography/mass spectrometry analysis of the pentafluorobenzyl esters, the following ions were used for quantification: $m / z 141\left(\mathrm{D}_{6}-\mathrm{SA} ; \mathrm{R}_{\mathrm{f}}=\right.$ $9.19 \mathrm{~min}), m / z 137\left(\mathrm{SA} ; \mathbf{R}_{\mathrm{f}}=9.22 \mathrm{~min}\right), m / z 215\left(\mathrm{D}_{6}-\mathrm{JA} ; \mathbf{R}_{\mathrm{f}}=\right.$ 14.12, $14.48 \mathrm{~min})$, and $\mathrm{m} / z 209\left(\mathrm{JA} ; \mathrm{R}_{\mathrm{f}}=14.17,14.53 \mathrm{~min}\right)$, respectively.

\section{ACKNOWLEDGMENTS}

We thank B. Mauch-Mani for providing the BMM1 strain of B. cinerea

\section{LITERATURE CITED}

Abe, H., Ohnishi, J., Narusaka, M., Seo, S., Narusaka, Y., Tsuda, S., and Kobayashi, M. 2008. Function of jasmonate in response and tolerance of Arabidopsis to thrips feeding. Plant Cell Physiol. 49:68-80.

Beckers, G. J. M., and Spoel, S. H. 2006. Fine-tuning plant defense signaling: Salicylate versus jasmonate. Plant Biol. 8:1-10.

Berger, S., Sinha, A. K., and Roitsch, T. 2007. Plant physiology meets phytopathology: Plant primary metabolism and plant-pathogen interactions. J. Exp. Bot. 58:4019-4026.

Boch, J., Verbsky, M. L., Roberston, T. L., Larkin, J. C., and Kunkel, B. N. 1998. Analysis of resistance gene-mediated defense responses in Arabidopsis thaliana plants carrying a mutation in CPR5. Mol. Plant-Microbe Interact. 12:1196-1206.

Bolton, M. D. 2009. Primary metabolism and plant defense. Mol. PlantMicrobe Interact. 22:487-497.

Chivasa, S., Murphy, A. M., Hamilton, J. M., Lindsey, K., Carr, J. P., and Slabas, A. R. 2009. Extracellular ATP is a regulator of pathogen defense in plants. Plant J. 60:436-448.

Conrath, U., Pieterse, C. M. J., and Mauch-Mani, B. 2002. Priming in plant-pathogen interactions. Trends Plant Sci. 7:210-216.

Conrath, U., Linke, C., Jeblick, W., Geigenberger, P., Quick, W. P., and Neuhaus, H. E. 2003. Enhanced resistance to Phytophthora infestans and Alternaria solani in leaves and tubers, respectively, of potato plants with decreased activity of the plastidic ATP/ADP transporter. Planta 19:75-83.

Conrath, U., Beckers, G. J. M., Flors, V., García-Agustín, P., Jakab, G., Mauch, F., Newman, M.-A., Pieterse, C. M. J., Poinssot, B., Pozo, M. J., Pugin, A., Schaffrath, U., Ton, J. Wendehenne, D., Zimmerli, L., and Mauch-Mani, B. 2006. Priming: Getting ready for battle. Mol. PlantMicrobe Interact. 19:1062-1071.

De Vos, M., Van Zaanen, W., Koornneef, A., Korzelius, J. P., Dicke, M., Van Loon, L. C., and Pieterse, C. M. J. 2006. Herbivore-induced resistance against microbial pathogens in Arabidopsis. Plant Physiol. 142:352-363.

Dong, X. 2004. NPR1, all things considered. Curr. Opin. Plant Biol. 7:547552.

Foresi, N. P., Laxalt, A. M., Tonón, C. V., Casalongué, C. A., and Lamattina, L. 2007. Extracellular ATP induces nitric oxide production in tomato cell suspensions. Plant Physiol. 145:589-592.

Jung, H.-S., and Chory, J. 2009. Signaling between chloroplasts and the nucleus: Can a systems biology approach bring clarity to a complex and highly regulated pathway? Plant Physiol. 152:453-459.

Kessler, A., and Baldwin, I .T. 2002. Plant responses to insect herbivory: The emerging molecular analysis. Annu. Rev. Plant Biol. 53:299-328.

Koornneef, A., and Pieterse, C. M. J. 2008. Crosstalk in defense signaling. Plant Physiol. 146:839-844.

Koornneef, A., Leon-Reyes, A., Ritsema, T., Verhage, A., Den Otter, F. C., Van Loon, L. C., and Pieterse, C. M. J. 2008. Kinetics of salicylatemediated suppression of jasmonate signaling reveal a role for redox modulation. Plant Physiol. 147:1358-1368.

Leon-Reyes, A., Spoel, S. H., De Lange, E. S., Abe, H., Kobayashi, M., Tsuda, S., Millenaar, F. F., Welschen, R. A. M., Ritsema, T., and Pieterse, C. M. J. 2009. Ethylene modulates the role of NONEXPRESSOR OF PATHOGENESIS-RELATED GENES 1 in crosstalk between salicylate and jasmonate signaling. Plant Physiol. 149:1797-1809.

Linka, N., Hurka, H., Lang, B. F., Burger, G., Winkler, H. H., Stamme, C., Urbany, C., Seil, I., Kusch, J., and Neuhaus, E. H. 2003. Phylogenetic relationship of non-mitochondrial nucleotide transport proteins in bacteria and eukaryotes. Gene 306:27-35.

Linke, C., Conrath, U., Jeblick, W., Betsche, T., Mahn, A., Duering, K., and Neuhaus, H. E. 2002. Inhibition of the plastidic ATP/ADP transporter protein primes potato tubers for augmented elicitation of defense responses and enhances their resistance against Erwinia carotovora. Plant Physiol. 129:1607-1615. 
Mauch-Mani, B., and Slusarenko, A. J. 1994. Systemic acquired resistance in Arabidopsis thaliana induced by a predisposing infection with a pathogenic isolate of Fusarium oxysporum. Mol. Plant-Microbe Interact. 7:378-383

Neuhaus, H. E., and Wagner, R. 2000. Solute pores, ion channels, and metabolite transporters in the outer and inner envelope membranes of higher plant plastids. Biochim. Biophys. Acta 1465:307-323.

Neuhaus, H. E., Thom, E., Möhlmann, T., Steup, M., and Kampfenkel, K. 1997. Characterization of a novel eukaryotic ATP/ADP translocator located in the plastid envelope of Arabidopsis thaliana. Plant J. 11:73-82.

Reinhold, T., Alawady, A., Grimm, B., Beran, K. C., Jahns, P., Conrath, U., Bauer, J., Reiser, J., Melzer, M., Jeblick, W., and Neuhaus, H. E. 2007. Limitation of nocturnal import of ATP into Arabidopsis chloroplasts leads to photooxidative damage. Plant J. 50:293-304.

Reiser, J., Linka, N., Lemke, L., Jeblick, W., and Neuhaus, H. E. 2004 Molecular physiological analysis of the two plastidic ATP/ADP transporters from Arabidopsis thaliana. Plant Physiol. 136:3524-3536.

Rethage, J., Ward, P. I., and Slusarenko, A. J. 2000. Race-specific elicitors from the Peronospora parasitica/Arabidopsis thaliana pathosystem. Physiol. Mol. Plant Pathol. 56:179-184.

Reymond, P., and Farmer, E. E. 1998. Jasmonate and salicylate as global signals for defense gene expression. Curr. Opin. Plant Biol. 1:404-411.

Song, C. J., Steinebrunner, I., Wang, X., Stout, S. C., and Roux, S. J. 2006 Extracellular ATP induces the accumulation of superoxide via NADPH oxidases in Arabidopsis. Plant Physiol. 140:1222-1232.

Stumpe, M., Carsjens, J.-G., Stenzel, I., Göbel, C., Lang, I., Pawlowski,
K., Hause, B., and Feussner, I., 2005. Lipid metabolism in arbuscular mycorrhizal roots of Medicago truncatula. Phytochemistry 66:781-791.

Thomma, B. P. H. J., Eggermont, K., Penninckx, I. A. M. A., Mauch-Mani, B., Vogelsang, R., Cammue, B. P. A., and Broekaert, W. F. 1998. Separate jasmonate-dependent and salicylate-dependent defense-response pathways in Arabidopsis are essential for resistance to distinct microbial pathogens. Proc. Natl. Acad. Sci. U.S.A. 95:15107-15111.

Ton, J., Van Pelt, J. A., Van Loon, L. C., and Pieterse, C. M. J. 2002. Differential effectiveness of salicylate-dependent and jasmonate/ethylenedependent induced resistance in Arabidopsis. Mol. Plant-Microbe Interact. 15:27-34

Tornero, P., and Dangl, J. L. 2001. A high-throughput method for quantifying growth of phytopathogenic bacteria in Arabidopsis thaliana. Plant J. 28:475-481.

Trentmann, O., Jung, B., Neuhaus, H. E., and Haferkamp, I. 2008. Nonmitochondrial ATP/ADP transporters accept phosphate as third substrate. J. Biol. Chem. 283:36486-36493.

Wu, S.-J., Liu, Y.-S., and Wu, J.-Y. 2008. The signaling role of extracellular ATP and its dependence on $\mathrm{Ca}^{2+}$ flux in elicitation of Salvia miltiorrhiza hairy root cultures. Plant Cell Physiol. 49:617-624.

Yu, I.-C., Parker, J., and Bent, A. F. 1998. Gene-for-gene disease resistance without the hypersensitive response in Arabidopsis dnd1 mutant. Proc. Natl. Acad. Sci. U.S.A. 95:7819-7824.

Zimmerli, L., Métraux, J.-P., and Mauch-Mani, B. 2001. $\beta$-Aminobutyric acid-induced protection of Arabidopsis against the necrotrophic fungus Botrytis cinerea. Plant Physiol. 126:517-523. 